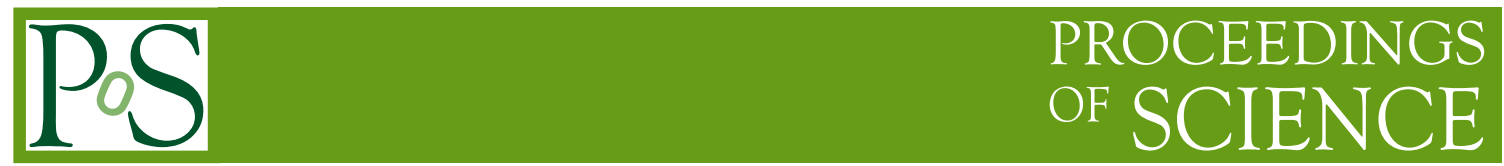

\title{
Prospects for Rare Decays at Belle II
}

\section{S. Sandilya*t}

University of Cincinnati, Cincinnati, Ohio 45221

E-mail: saurabhsandilya@gmail.com

Belle II is an upcoming experiment at the next generation SuperKEKB $e^{+} e^{-}$collider. It will record about $50 \mathrm{ab}^{-1}$ of data mostly at the $\Upsilon(4 S)$ resonance. The high statistics will allow precision measurements for rare decays, which can be then compared with the Standard Model predictions in order to search indirectly for new physics. We present the prospects for rare decay studies at Belle II.

9th International Workshop on the CKM Unitarity Triangle

28 November - 3 December 2016

Tata Institute for Fundamental Research (TIFR), Mumbai, India

* Speaker.

${ }^{\dagger}$ On behalf of the Belle II Collaboration 


\section{Introduction}

B-factories had a successful operational period with a total recorded sample of over $1.5 \mathrm{ab}^{-1}$ of data corresponding to $1.2 \times 10^{9} \mathrm{~B}$-meson pairs. As a next generation B-factory, the Belle II experiment will search for new physics (NP) beyond the Standard Model (BSM). The Belle II detector will be located at the upgraded SuperKEKB $e^{+} e^{-}$asymmetric collider. It will record most of its data at the $\Upsilon(4 S)$ resonance, which gives a very clean sample of quantum correlated B-meson pairs. Belle II is expected to increase the data-sample size by a factor of 50 compared to Belle. The SuperKEKB accelerator has been designed to give an instantaneous luminosity of $8 \times 10^{35} \mathrm{~cm}^{-2} \mathrm{~s}^{-1}$, which is about 40 times larger than the previous KEKB accelerator. The first data taking run for physics analyses is expected to begin in early 2018. In this proceedings we present the prospects for rare decay studies at Belle II.

\section{Inclusive $B \rightarrow X_{s, d} \gamma$}

The $b \rightarrow(s, d) \gamma$ transition is a flavor changing neutral current (FCNC) process forbidden at tree level in the Standard Model (SM) and proceeds via a radiative 'penguin' loop diagram. These types of decays are sensitive to potential contributions from non-SM particles. The NP scenarios can be investigated through a precise measurement of the branching fraction (BF) of the inclusive $B \rightarrow X_{s, d} \gamma$ decays. The BF is predicted very precisely in the SM with $\mathscr{B}_{s \gamma}=(3.36 \pm 0.23) \times$ $10^{-4}$ and $\mathscr{B}_{d \gamma}=\left(1.73_{-0.22}^{+0.12}\right) \times 10^{-5}$, for $E_{\gamma}>1.6 \mathrm{GeV}$ [1]. Recently, Belle measured the BF for $B \rightarrow X_{s} \gamma$ process, using a fully inclusive method and the preliminary result (extrapolated for $\left.E_{\gamma}>1.6 \mathrm{GeV}\right) \mathscr{B}_{s \gamma}=(3.12 \pm 0.10$ (stat.) \pm 0.19 (sys.) \pm 0.08 (model) \pm 0.04 (extrap. $\left.)\right) \times 10^{-4}$ is the world's most precise measurement, which is in agreement with the SM prediction as well as previous measurements [2]. Evaluation of the constraint on BSM scenario depends crucially on both the central value and the uncertainties on the BF. The above mentioned Belle result with a fully inclusive method has $7.3 \%$ uncertainty and excludes a mass of the charged Higgs boson below $580 \mathrm{GeV}$ at $95 \%$ confidence level.

Belle II is expected to reduce the systematic uncertainty in the measurement with its large data sample. Conservatively estimated, $3.9 \%$ total error will be achievable with $50 \mathrm{ab}^{-1}$; see the projection plot in Figure 1 (left). This is comparable to the theory uncertainty due to nonperturbative effects (which is hard to reduce) [1]. In addition, at Belle II it will be also possible to measure the BF with $E_{\gamma}>1.6 \mathrm{GeV}$, then there won't be any need for extrapolation to compare the results with theoretical predictions.

In addition to the $\mathrm{BFs}$, isospin and $\mathrm{CP}$ asymmetries in the decay rates are also sensitive to the BSM contributions. The uncertainty on the isospin measurements can be improved at Belle II with more statistics. The SM predicts quite different $\mathrm{CP}$ asymmetries for $B \rightarrow X_{s} \gamma$ and $B \rightarrow X_{d} \gamma$, however for the sum of these decays it is predicted to be very small (close to zero, due to the unitarity of the CKM matrix). Further, the difference of $A_{C P}\left(B \rightarrow X_{s} \gamma\right)$ between charged and neutral B mesons $\left(\triangle A_{C P}\right)$ is sensitive to phases in Wilson coefficients $C_{7}$ and $C_{8}$, which is zero. So, if either $A_{C P}\left(B \rightarrow X_{s+d} \gamma\right)$ or $\Delta A_{C P}$ is deviated from zero, it will be a clear NP signal [3, 4]. In asymmetry measurements, most of the systematic errors cancel out, so both will be still statistically dominated at Belle II with $50 \mathrm{ab}^{-1}$. The uncertainties in $A_{C P}\left(B \rightarrow X_{s+d} \gamma\right)$ and $\Delta A_{C P}$ are projected to be 
$\pm 0.61 \%$ and $\pm 0.37 \%$, respectively. The Belle II projection plots for $A_{C P}$ and $\Delta A_{C P}$ are shown in Figure 1 (right).
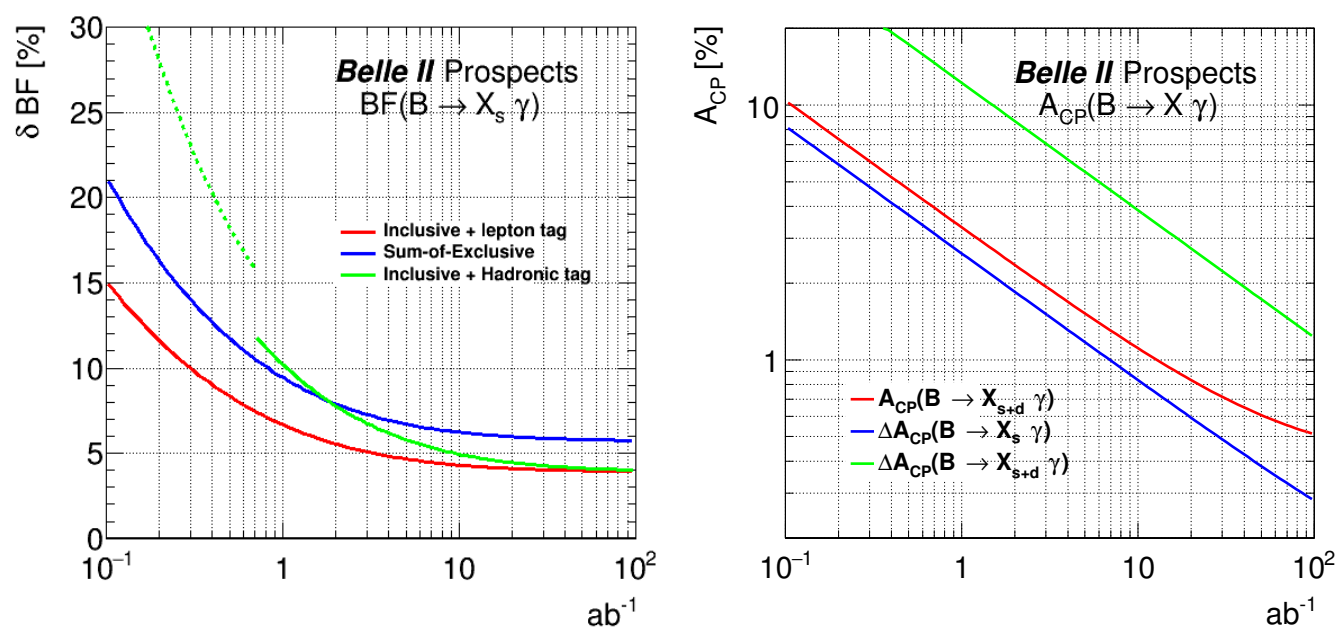

Figure 1: Belle II projections for uncertainities in inclusive $\mathscr{B}\left(B \rightarrow X_{s} \gamma\right)$ and $A_{C P}(B \rightarrow X \gamma)$.

\section{Exclusive $b \rightarrow s \gamma$}

Mixing-induced CP asymmetry in an exclusive $b \rightarrow s \gamma$ CP eigenstate mode such as $B \rightarrow$ $K^{\star}\left[K_{s}^{0} \pi^{0}\right] \gamma$ is an excellent probe for a particular class of NP scenario [5]. In the SM, expected asymmetry is $S_{K^{\star}\left[K_{s}^{0} \pi^{0}\right] \gamma}^{S M}=-(2.3 \pm 1.6) \%$ and $S_{\rho^{0}\left[\pi^{+} \pi^{-}\right] \gamma}^{S M}=-(0.2 \pm 1.6) \%[6,7,8]$. As the two final states from $B^{0}$ and $\overline{B^{0}}$ decays have photons of different helicity (opposite helicity photon is suppressed by the $m_{s} / m_{b}$ factor) and therefore do not mix. NP contribution with the right handed current would increase the fraction of right handed photon, and its interference with the SM can give large time dependent $\mathrm{CP}$ violation. Studies of these asymmetries are thus considered to be one of the most promising methods to search for the BSM right-handed currents. At Belle II, the vertex detector is larger than at Belle $\left(11.5 \mathrm{~cm}\right.$ radius in Belle II $c f .6 \mathrm{~cm}$ ), which will give $30 \%$ more $K_{s}^{0}$ with vertex hits. And also, effective tagging efficiency is $13 \%$ larger than at Belle (conservative estimation), hence significant improvement in the determination of $A_{C P}(t)$ in $B \rightarrow K^{\star}\left[K_{s}^{0} \pi^{0}\right] \gamma$ is expected. The Belle II projection plot for uncertainty in $A_{C P}(t)$ is shown in Figure 2 (left). With the Belle II projected uncertainty, the central value measured by Belle [9] would be $16 \sigma$ deviation from zero, as shown in Figure 2 (right).

\section{4. $b \rightarrow s \ell^{+} \ell^{-}$}

The $b \rightarrow s \ell^{+} \ell^{-}$is also an FCNC process, which is sensitive to NP. Owing to lepton universality in the SM, the ratio between the BFs of the electron mode to the muon mode is expected to be unity. LHCb reported $2.6 \sigma$ deviation of $R_{K}$ (ratio of the BFs between $B^{+} \rightarrow K^{+} \mu^{+} \mu^{-}$and $B^{+} \rightarrow$ $K^{+} e^{+} e^{-}$) from the SM expectation for low $q^{2}$ region [10]. Since the reconstruction of electron modes at Belle II is easier than at $\mathrm{LHCb}$ and have a comparable efficiency to that for muon modes. 

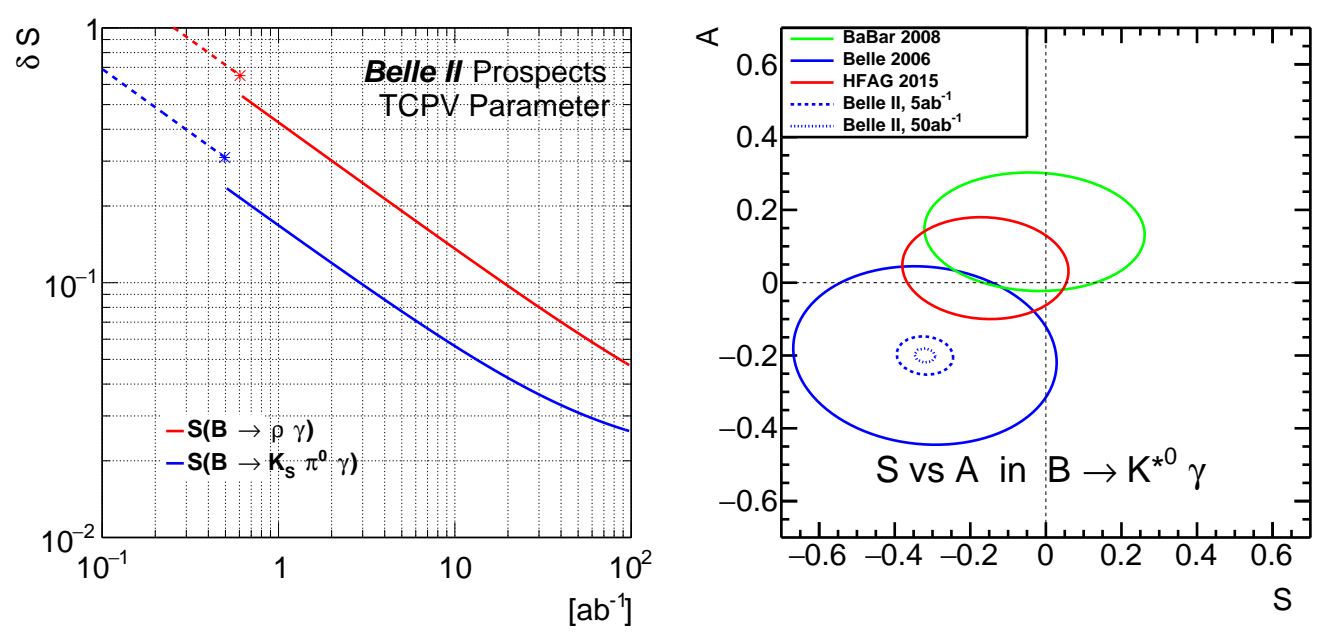

Figure 2: Belle II projections for uncertainities in inclusive $\mathscr{B}\left(B \rightarrow X_{s} \gamma\right)$ and $A_{C P}(B \rightarrow X \gamma)$.

The ratios $R_{K}, R_{K^{\star}}$ and $R_{X_{S}}$ can be measured precisely in Belle II for both low and high $q^{2}$ regions. In ratio measurements most of the systematic error cancels out except for lepton identification, which is expected to be about $0.4 \%$. The error will be mostly statistical dominant even with the Belle II data.

Measurement in the inclusive $B \rightarrow X_{s} \ell^{+} \ell^{-}$channel is theoretically cleaner than the exclusive channels, especially for $q^{2}$ region below the charm resonances [11, 12, 13, 14]. The measurements for $B \rightarrow X_{s} \ell^{+} \ell^{-}$decays in BaBar $[15,16]$ and Belle [17] suffer from a sizeable experimental uncertainties. Furthermore, these measurements are based on a sum over several exclusive states, which makes a direct comparison to the theoretical predictions difficult. It is expected from Belle II to improve upon the present situation. Belle has performed the first measurement of the forwardbackward asymmetry $\left(A_{F B}\right)$ in $B \rightarrow X_{s} \ell^{+} \ell^{-}$with the sum of several exclusive modes [18]. The $A_{F B}$ is found to be mostly consistent with the theoretical prediction [19] with a mild tension in the low $q^{2}$ region. The measurement of $A_{F B}$ can also be improved at Belle II, where the errors are projected to be in order of few $\%$ with the $50 \mathrm{ab}^{-1}$ data.

In order to provide a meaningful insight to the impact of $\mathrm{BF}$ and $A_{F B}$ measurements for $B \rightarrow X_{s} \ell^{+} \ell^{-}$in Belle II, we look at the potential for model-independent constraints on the relevant Wilson-coefficients derived from inclusive measurements only. Such projection is shown in Figure 3, considering the Wilson coefficients $C_{9}$ and $C_{10}$ as the ones potentially receiving relevant NP contributions [20].

\section{5. $b \rightarrow s v \bar{v}$}

The $b \rightarrow s v \bar{v}$ decays are the theoretically cleanest among the FCNC processes [21]. The BF of the decay $B \rightarrow K^{(\star)} v \bar{v}$ is mainly limited by $B \rightarrow K^{(\star)}$ form factors and relevant CKM matrix elements. The SM predictions are available in Ref. [21] and recently updated in Ref. [22]. Belle very recently updated $b \rightarrow(s, d) v \bar{v}$ BF measurement with semileptonic tagging [23]. These decays 


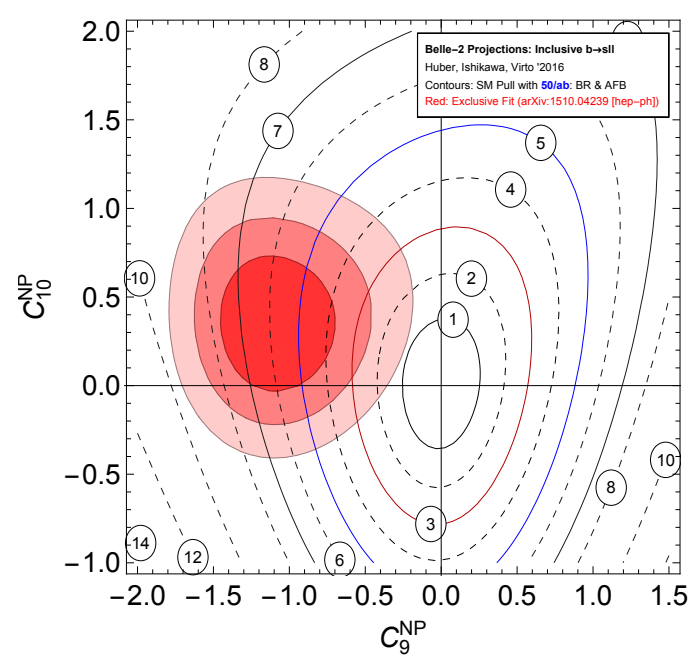

Figure 3: Potential of inclusive $b \rightarrow s \ell^{+} \ell^{-}$measurements at Belle II with current exclusive constraints [20].

can be observed with Belle II, assuming the SM prediction holds. Belle II will be able to provide a measurement with uncertainties of similar size as the current theoretical uncertainties.

\section{Summary and Status}

Belle II has a rich physics program; both complementary to, and competitive with, the LHCb experiment and energy frontier flavor physics programs. With the more powerful Belle II detector and higher luminosity machine SuperKEKB, we can search with high statistics for NP in radiative and electroweak penguin decays. Accelerator commissioning (phase 1) was successful in June 2016. Phase 2 without vertex detector will start in the end of 2017 and phase 3 with the complete detector is expected in the fall of 2018. The Belle II detector is now mostly installed and currently being commissioned for the phase 2 running.

\section{References}

[1] M. Misiak et al., Phys. Rev. Lett. 114, 221801 (2015).

[2] A. Abdesselam et al. (Belle Collaboration), arXiv:1608.02344.

[3] T. Hurth, E. Lunghi, and W. Porod, Nucl. Phys. B704, 56 (2005).

[4] M. Benzke, S.J. Lee, M. Neubert, and G. Paz, Phys. Rev. Lett. 106, 141801 (2011).

[5] D. Atwood, M. Gronau, and A. Soni, Phys. Rev. Lett. 79, 185 (1997).

[6] P. Ball, G.W. Jones, and R. Zwicky, Phys. Rev. D 75, 054004 (2007).

[7] P. Ball, and R. Zwicky, Phys. Lett. B 642, 478 (2006).

[8] A. Bharucha, D. M. Straub, and R. Zwicky, JHEP 08, (2016) 098.

[9] Y. Ushiroda et al. (Belle Collaboration), Phys. Rev. D 74, 111104(R) (2006).

[10] R. Aaij et al. (LHCb Collaboration), Phys. Rev. Lett. 113, 151601 (2014). 
[11] C. Bobeth, P. Gambino, M. Gorbahn, and U. Haisch, JHEP 04, 071 (2004).

[12] T. Huber, E. Lunghi, M. Misiak, and D. Wyler, Nucl. Phys. B740, 105 (2006).

[13] T. Huber, T. Hurth, and E. Lunghi, Nucl. Phys. B802, 40 (2008).

[14] T. Huber, T. Hurth, and E. Lunghi, JHEP 06, 176 (2015).

[15] B. Aubert et al. (BaBar Collaboration), Phys. Rev. Lett. 93, 081802 (2004).

[16] J. P. Lees et al. (BaBar Collaboration), Phys. Rev. Lett. 112, 211802 (2014).

[17] M. Iwasaki et al. (Belle Collaboration), Phys. Rev. D 72, 092005 (2005).

[18] Y. Sato et al. (Belle Collaboration), Phys. Rev. D 93, 032008 (2016).

[19] S. Fukae, C. S. Kim, T. Morozumi, and T. Yoshikawa, Phys. Rev. D 59, 074013 (1999).

[20] T. Huber, A. Ishikawa, and J. Virto (private communication), to be published in B2TiP report.

[21] A. J. Buras, J. Girrbach-Noe, C. Niehoff, and D. M. Straub, JHEP 02, 184 (2015).

[22] D. M. Straub, BELLE2-MEMO-2016-007 (2016).

[23] J. Grygier et al. (Belle Collaboration), arXiv:1702.03224 